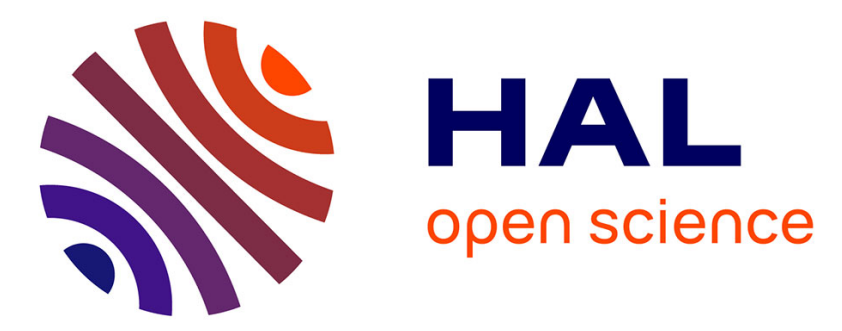

\title{
Understanding water effect on Candida antarctica lipase $B$ activity and enantioselectivity towards secondary alcohols.
}

\author{
Valérie Léonard, Z. Marton, Sylvain Lamare, Karl Hult, Marianne Graber
}

\section{- To cite this version:}

Valérie Léonard, Z. Marton, Sylvain Lamare, Karl Hult, Marianne Graber. Understanding water effect on Candida antarctica lipase B activity and enantioselectivity towards secondary alcohols.. Journal of Molecular Catalysis B: Enzymatic, 2009, 59, pp.90-95. 10.1016/j.molcatb.2009.01.008 . hal-00647583

\section{HAL Id: hal-00647583 \\ https://hal.science/hal-00647583}

Submitted on 2 Dec 2011

HAL is a multi-disciplinary open access archive for the deposit and dissemination of scientific research documents, whether they are published or not. The documents may come from teaching and research institutions in France or abroad, or from public or private research centers.
L'archive ouverte pluridisciplinaire HAL, est destinée au dépôt et à la diffusion de documents scientifiques de niveau recherche, publiés ou non, émanant des établissements d'enseignement et de recherche français ou étrangers, des laboratoires publics ou privés. 


\section{Understanding water effect on Candida antarctica lipase B activity and enantioselectivity towards secondary alcohols}

Valérie Léonard-Neversa ${ }^{\text {a }}$ Zsuzsanna Martona, Sylvain Lamare ${ }^{\mathrm{a}}$, Karl Hult $^{\mathrm{b}}$, Marianne Graber ${ }^{\mathrm{a}, *}$

${ }^{a}$ Université de la Rochelle, Pôle Sciences- Bât. Marie Curie, UMR6250 LIENSs CNRS-ULR, Avenue Michel Crépeau, 17042 La Rochelle, Cedex 01, France.

b Department of Biochemistry, School of Biotechnology, Royal Institute of Technology, AlbaNova University Center, SE-106 91 Stockholm, Sweden.

*Corresponding author: Phone: +33 546458630 Fax: +33 54645 8265, E-mail address: mgraber@univ-lr.fr 


\begin{abstract}
The effect of water activity $\left(\mathrm{a}_{\mathrm{W}}\right)$ on Candida antarctica lipase B (CALB) activity and enantioselectivity towards secondary alcohols was assessed. Experimental results for the resolution of racemic pentan-2-ol, hexan-3-ol, butan-2-ol and octan-4-ol by immobilized CALB-catalyzed acylation with methyl propanoate, were obtained by using a solid/gas reactor. Water and substrate adsorption mechanism on immobilized CALB, were then studied using moisture sorption analyzer and inverse gas chromatography, and the effective hydration state of the biocatalyst when varying $\mathrm{a}_{\mathrm{w}}$ was defined. The data showed a pronounced $\mathrm{a}_{\mathrm{w}}$ effect on both activity and enantioselectivity. If secondary alcohol follows the steric rules for being efficiently resolved, water at very low aw increased enantioselectivity by acting predominantly as an enantioselective inhibitor, making the stereospecificity pocket smaller. When increasing $\mathrm{a}_{\mathrm{W}}$, water decreased enantioselectivity, due to an unfavourable increase of the entropic term $T \Delta_{\mathrm{R}-\mathrm{S}} \Delta \mathrm{S}^{\ddagger}$ of the differential free energy of activation. The "turning point" at which water changed from one predominant role to another would correspond to $\mathrm{a}_{\mathrm{W}}$ allowing full coverage of polar groups of the immobilized biocatalyst by water molecules.
\end{abstract}

Keywords: lipase; stereoselective catalysis; thermodynamics; thermodynamic activity of water; water adsorption 


\section{Introduction}

Enantioselectivity is an extremely important property of lipases and is the basis of numerous applications for the preparation of optically pure alcohols and esters. Although the fast reacting enantiomer can be foreseen using empirical rules [1], the magnitude of the enantioselectivity can seldom be predicted. The molecular basis of chiral recognition is complex and not completely elucidated, in spite of numerous efforts for setting up rules and criteria of enantioselectivity, based both on experimental results and molecular modelling studies. In particular, previous studies have shown that the effect of substrate structure [1-4], solvent of reaction [5-7], thermodynamic activity of water $\left(\mathrm{a}_{\mathrm{w}}\right)[6-9]$ and temperature $[10,11]$ on enantioselectivity, is complex and still needs a better molecular understanding.

In a previous paper [12] we have shown that water has many effects on Candida antarctica lipase B (CALB) catalyzed enantioselective reactions, as water could be simultaneously a competitive and enantioselective inhibitor, a competitive substrate and a lubricant. These results were obtained by combining molecular modelling studies and experimental work performed in a solid/gas reactor, which allows precise control of the thermodynamic parameters of the enzyme microenvironment, and permits the determination of the specific role of water addition [13].

In the present paper, the influence of water on enantioselectivity of CALB is further investigated for a series of linear secondary alcohols. The effect of $\mathrm{a}_{\mathrm{W}}$ on activity and selectivity of CALB was experimentally measured using a solid/gas reactor. Moreover, these results were completed by studies of the adsorption mechanism of water and substrate molecules on immobilized CALB. The idea was to correlate the different effects of water on CALB enantioselectivity with the effective hydration of the catalyst. To perform these adsorption studies, we chose a dynamic and very accurate vapour adsorption technique: Inverse Gas Chromatography (IGC). The term "Inverse Gas Chromatography” was introduced 
to denote that the interest is in the stationary phase in the column, which was the immobilized CALB in our case. IGC uses gaseous probes which are injected in the column, to determine their interactions with the solid in the column. Quantization of the interaction between a given probe and the solid in the column is achieved by the determination of the retention time of the probe. Furthermore, IGC experiments may be carried out over appreciable temperature ranges, so that the temperature dependence of interactions can be determined. These data provide thermodynamic information and adsorption properties of the support, like surface energy of adsorption $[14,15]$.

Our goal has been to further expand the knowledge on how the enzyme solves the task of distinguishing between enantiomers of secondary alcohols and to understand the variations in enthalpy and entropy on a molecular level when varying aw.

\section{Experimental}

\subsection{Enzyme and chemicals}

C. antarctica lipase B was produced and purified in house [16] or was a lyophilized preparation (Chirazyme L2) kindly supplied by Roche Industrie (Penzberg, Germany). All substrates used in solid/gas reactor experiments and probes used in IGC experiments were of the highest purity (98\% minimum) and checked by gas chromatography before use. Chemicals were dried by distillation under argon prior to use. Substrates were purified and stored under argon atmosphere and over molecular sieves. (R,S)-2-butylpropanoate, (R,S)-2pentylpropanoate, $(\mathrm{R}, \mathrm{S})$-3-hexylpropanoate, and (R,S)-4-octylpropanoate were synthesized from butan-2-ol, pentan-2-ol, hexan-3-ol, or octan-4-ol and propanoic acid in the presence of p-toluene sulfonic acid. 


\subsection{Adsorption of lipase onto a solid support}

For solid/gas experimental studies, CALB adsorption was performed onto 60-80 mesh chromosorb P AW DMCS, (Acid Washed DiMethylChloroSilanized, Varian, France), as previously described [12]. For water and organic probes adsorption studies, enzyme adsorption was performed by using $30 \mathrm{mg}$ of lyophilized Chirazyme L2 per $6 \mathrm{~g}$ of support. Both preparations of immobilized CALB had the same enzymatic activity per mg of support.

\subsection{Experimental setup for solid-gas catalysis}

The bioreactor used in this study has been described in a previous publication [13] The packed-bed bioreactor was a glass tube (length $9 \mathrm{~cm}, 6 \mathrm{~mm}$ o.d., $4 \mathrm{~mm}$ i.d.) in which a known mass of the enzymatic preparation was packed, between two layers of glass wool. Substrate or solvent feeding was achieved by passing dried nitrogen, as carrier gas, through the substrate/solvent flasks. Substrates and solvent were continuously passed through the bioreactor to react with the lipase. Thermodynamic activities of the compounds in the reactor were defined as the ratio of their partial pressure in gas entering the bioreactor to their saturation pressure at the working temperature. These were obtained by adjusting the volumetric flow rates of the carrier gases in the different lines, according to the calculations explained previously [13]. Calculations were performed by assuming that the gas was close to ideal (fugacity was not taken into account). The gas leaving the bioreactor was injected into a gas chromatograph for analysis. All the parameters (volumetric flow rates, molar flow rates, substrate and solvent thermodynamic activities, partial pressures, temperatures) were monitored on-line using a computer and software was developed for the automation and the regulation of the running conditions.

Experiments were run with immobilized enzyme preparation (varied from $10 \mathrm{mg}$ to $600 \mathrm{mg}$ depending on the substrate used) diluted (8 times) with dried chromosorb P AW DMCS in 
order to obtain initial rate conditions. It was checked that the addition of dried support to the adsorbed enzyme had no influence on the enantioselectivity. The total flow rate passing through the reactor was set from 0.25 to $1.5 \mathrm{mmol} \mathrm{min}^{-1}$ depending on the substrate used. For activity and enantioselectivity measurements, thermodynamic activities of substrates were fixed at 0.05 for the alcohol and 0.1 for methyl propanoate. Temperature was fixed at $318 \mathrm{~K}$ for activity and enantioselectivity measurements. For determination of thermodynamic components of enantioselectivity, the temperature range was 313 to $338 \mathrm{~K}$. In these conditions, conversion level of substrates was comprised between 5 and $10 \%$.

\subsection{Chromatographic assays}

For the solid/gas system analyses, the vapour phase leaving the bioreactor was sampled by using a loop $(0.25 \mathrm{ml})$ on a six-way valve (Valco) maintained at $175^{\circ} \mathrm{C}$. Samples were automatically injected in the split injector of a gas chromatograph (Agilent model 6890N Series) equipped with a flame ionization detector (FID) for detection of all products. The column used was a Chirasil-Dex CB $(25 \mathrm{~m}$ x $0.25 \mathrm{~mm}$ i.d. x $0.25 \mu \mathrm{m} \beta$-cyclodextrin; Chrompack, France). The injector and the detector were kept at $250^{\circ} \mathrm{C}$. Nitrogen was used as the carrier gas with a constant flow rate $(1.8 \mathrm{~mL}$ min-1). Hydrogen and air were supplied to the FID at 35 and $350 \mathrm{~mL}$ min-1, respectively). Quantitative data were obtained by integration on an Agilent 3396 Series III integrator.

The external calibration of the 5 substrates (butan-2-ol, pentan-2-ol, hexan-3-ol, octan-4-ol and methyl propanoate) was carried out by programming a range of their partial pressures in the bioreactor, and by gas chromatography analysis. For the products (2-butylpropanoate, 2pentylpropanoate, 3-hexylpropanoate, 4-octylpropanoate and methanol), an internal calibration was carried out by using respectively butan-2-ol, pentan-2-ol, hexan-3-ol, octan-4ol and methyl propanoate as internal standards. 
Depending on the substrate studied, for accurate determination of E-values the vapour phase leaving the bioreactor was condensed for a period of time depending on the molar flow rates, and then partially evaporated in order to enhance the detection and quantification of the

products. Then unreacted methyl propanoate (boiling point $78-79^{\circ} \mathrm{C}$ ) was then eliminated and this enrichment of reaction product enabled an accurate measurement of the enantiomeric ratio [12]. Elution peaks of (R)-alcohol and (S)-alcohol were identified using pure commercial (R)-alcohol. The absolute configuration of the reaction products was established by esterification of commercial pure alcohol enantiomers.

\subsection{Determination of the enantiomeric ratio and of thermodynamic activation parameters}

The enantiomeric ratio, E was determined according to the method described in a previous publication [17]. The following equations

$\mathrm{RT} \ln \mathrm{E}=-\Delta_{\mathrm{R}-\mathrm{S}} \Delta G^{t}$,

and $\Delta_{\mathrm{R}-\mathrm{S}} \Delta G^{t}=\Delta_{\mathrm{R}-\mathrm{S}} \Delta H^{*}-\mathrm{T} \Delta_{\mathrm{R}-\mathrm{S}} \Delta S^{*}$

were used to calculate the thermodynamic activation parameters [10, 11].

\subsection{Measurement of biocatalyst water adsorption isotherm}

Water adsorption isotherm of Chromosorb alone and with immobilized lipase were measured at $25^{\circ} \mathrm{C}$ by using a DVS Advantage automated moisture sorption analyzer (Surface Measurement Systems Ltd., London, U.K.) between 0 and 95\% relative humidity. The samples were initially dried for 10 hours at $25^{\circ} \mathrm{C}$ under flowing air to obtain the dry sample mass.

\subsection{Inverse Gas Chromatography (IGC) measurements}

Experiments were performed using an SMS-IGC2000 apparatus (Surface Measurement Systems Ltd., London, U.K.), allowing fully automated determination of interactions of ten 
different gas probe molecules with solid column packing material under investigation (Chromosorb with immobilized CALB in our case), under a wide range of humidity and temperature conditions. A complete explanation of the design of this apparatus can be found in reference [18].

To determine the enthalpies of adsorption ( $\triangle H a d s$ ) of pentan-2-ol on immobilized CALB, DMCS treated 4mm ID glass column packed with $530 \mathrm{mg}$ of immobilized CALB was used. Before measurements, column packed with immobilized biocatalyst was pre-conditioned for 10 hours at the initial temperature and $\mathrm{a}_{\mathrm{w}}$ studied. An aliquot of $250 \mu \mathrm{l}$ of pentan-2-ol were then injected at varying temperature between 308 and $324 \mathrm{~K}$ for $0<\mathrm{a}_{\mathrm{w}}<0.15$ and between 314 and $322 \mathrm{~K}$ for higher $\mathrm{a}_{\mathrm{w}}$ values. Pentan-2-ol vapour concentration in the carrier gas was such that $\mathrm{p} / \mathrm{p}^{\circ}=0.05$, with $\mathrm{p}$ and $\mathrm{p}^{\circ}$ being respectively equal to pentan-2-ol partial pressure in the gas and pentan-2-ol saturated vapour pressure, calculated by assuming an ideal gas type behaviour by $\mathrm{p}^{\circ}(\mathrm{T})=\mathrm{e}^{(-\Delta \mathrm{Hvap} / \mathrm{RT})}$. The carrier gas (helium) flow rate was $10 \mathrm{ml} / \mathrm{min}$. The net retention volume $V_{N}$ was then calculated using Eq (1), where $t$ (min) is the retention time of pentan-2-ol, $t_{0}(\mathrm{~min})$ is the dead-time obtained with methane, $F\left(\mathrm{ml} \cdot \mathrm{min}^{-1}\right)$ the carrier gas flow rate, $m(\mathrm{~g})$ the mass of immobilized CALB and $T(\mathrm{~K})$ the temperature of the column. The James and Martin compressibility factor, $j$, was calculated using Eq (2), where $P_{i}$ and $P_{0}$ are the inlet and outlet pressures, respectively.

$$
\begin{aligned}
& V_{N}=j \cdot \frac{\left(t-t_{0}\right)}{m} \cdot \frac{F \times 273.15}{T} \\
& j=\frac{3}{2} \cdot \frac{\left(P_{i} / P_{0}\right)^{2}-1}{\left(P_{i} / P_{0}\right)^{3}-1}(2)
\end{aligned}
$$

IGC is a reliable method to obtain heat of sorption values of gaseous compounds on solid support directly from their net retention volumes. To perform such measurement, IGC is used at infinite dilution, which means that a very small amount of probe vapour is injected into the carrier gas stream through the column. The retention volume of the probe is thus independent 
of its concentration and the result is a linear adsorption isotherm, which can be described by Henry's Law. Under these conditions, $V_{N}$ is related to the differential heat of sorption ( $\left.\Delta H a d s\right)$ by $\mathrm{Eq} 3$ :

$\ln \left(V_{N} / T\right)=-\Delta H_{a d s} / R T+C$

where it is assumed that the adsorption enthalpy is independent of temperature. $\Delta H a d s$ is then obtained from a plot of $\ln \left(V_{N} / T\right)$ versus $1 / T . \mathrm{Eq}(3)$ is derived from the Clausius-Clapeyron equation $\left(\frac{\partial \ln p}{\partial(1 / T))_{v}}=-\frac{\Delta H_{s t}}{R}\right.$, giving the differential isosteric heat of adsorption $\Delta \mathrm{H}_{\mathrm{st}}$, by plotting ln $\mathrm{p}$ versus $1 / \mathrm{T}$, applied to chromatographic data at infinite dilution, which are necessarily isosteric (constant adsorbate coverage of zero) [15].

\section{Results and discussion}

\subsection{Water activity and reaction rates}

Among the four linear secondary alcohols studied, the most efficient nucleophiles for acyl transfer reaction with methyl propanoate were those having a methyl as medium-sized substituent at the stereocenter (pentan-2-ol and butan-2-ol). The acyl transfer activity decreased significantly (by a factor 10), when this group became an ethyl (hexan-3-ol), and dramatically (by a factor 1000) when it was a propyl (octan-4-ol) (Figure 1). This is in accordance with the structural model of CALB [19] revealing the existence of a stereospecificity pocket in the active site of CALB, which harbours one of the substituents at the stereocenter of $s e c$-alcohols, as long as it is smaller than $n$-propyl. As a consequence the reaction rate is significantly affected by the size of the medium-sized substituent. Acylation is faster with pentan-2-ol than butan-2-ol, showing that the length of the large substituent at the stereocenter also has an influence on reaction rate. 
Concerning acylation reaction rate, the effect of water as lubricant is visible for pentan-2-ol and hexan-3-ol, with an increased activity in the range going from $\mathrm{a}_{\mathrm{W}}$ values near to 0 up to 0.02. For all secondary alcohols, acylation reaction rate decreased when aw increased further, as water acted as a competitive nucleophile of alcohols (Figure 1).

Hydrolysis activity in the absence of any alcohol increased with $\mathrm{a}_{\mathrm{w}}$ up to $\mathrm{a}_{\mathrm{w}}$ equal to 0.3 where the activity reached a plateau. In the presence of the fast reacting alcohols (pentan-2-ol and butan-2-ol) a steady increase of hydrolysis was seen but not reaching the same activity as was seen without alcohols, while much lower hydrolysis rates were reached in the presence of hexan-3-ol and octan-4-ol (Figure 2). As a result, total enzyme activity decreased with $\mathrm{a}_{\mathrm{W}}$ for all alcohols except for butan-2-ol (Figure 3). This has to be related to previous results showing that water and alcohols form a dead-end complex with free CALB, playing the role of competitive inhibitors of the first substrate methyl propanoate [20,21].

\subsection{Water activity and enantioselectivity}

The enantioselectivity is high in the total range of aw studied for pentan-2-ol and hexan-3-ol, reaching maxima of 320 and 120, respectively, whereas it is dramatically lower for butan-2-ol and octan-4-ol, 9 and 7 respectively (Figure 4). These experimental results fully support the model proposed for molecular recognition of sec-alcohol enantiomers by CALB [2]. According to this model, the preferred $(R)$-enantiomer reacts with its large substituent of the stereocenter orientated towards the entrance of the active site, while it places its medium group in a site of limited size, the stereoselectivity pocket. The slow enantiomer has to position its large substituent in the stereoselectivity pocket. Pentan-2-ol and hexan-3-ol both have a large substituent (propyl) hardly fitting in the pocket and a medium one (respectively methyl and ethyl) easily doing this, explaining the high enantioselectivity. The higher selectivity towards pentan-2-ol compared to hexan-3-ol is afforded by a larger difference 
between large and medium groups. In the case of butan-2-ol, the pocket can accommodate either the medium or the large substituent, whereas for octan-4-ol both substituents are too big for good binding, which leads in both cases to a very low selectivity. A drop in enantioselectivity of CALB towards secondary alcohols, when the medium substituent is extended over n-propyl has already been mentioned [2].

The increase in enantioselectivity at low water activity for pentan-2-ol has in an earlier paper been attributed to a water molecule that specifically binds in the stereoselectivity pocket and thereby reduces its size. The reduced size of the pocket enhances the difference in binding of the substituents at the stereocenter of the alcohol and thereby increases the enantioselectivity [12]. In Figure 4A it can be seen that hexan-3-ol had a similar increase at low water activity. Furthermore, using the Hill equation we could show that the water molecule trapped in the stereoselectivity pocket had a dissociation constant of 0.035 , corresponding to a binding energy of $9 \mathrm{~kJ} \mathrm{~mol}^{-1}$ in excellent agreement with the pentan-2-ol case.

Applying similar calculations in the water activity range 0.05-0.2 it can be hypothesized that two water molecules were bound in the stereoselectivity pocket. That would restrict the size of substituents that can occupy the pocket to methyl. This is in good agreement with the increase in enantioselectivity for butan-2-ol and pentan-2-ol. The enantioselectivity towards hexan-3-ol and octane-4-ol having larger medium sized substituents would not benefit from this further decrease in volume of the stereoselectivity pocket and accordingly did not show the same increase. Further increase of water activity would allow even more water molecules to bind to the stereoselectivity pocket. Reaching this point any secondary alcohol must expel water molecules from the binding site to be able to react. Secondary alcohols with small medium sized substituents would then not be favoured as much as at lower water activities. The difference between enantiomers would decrease and with that enantioselectivity. 
These last explanations of enantioselectivity at different $a_{w}$ rely only on steric differences between transition states of enantiomers, which correspond to differences in activation enthalpy and not entropy. Nevertheless thermodynamic analysis of enantioselectivity has revealed that entropy as well, is an important parameter for enzyme discrimination between enantiomers [22-24].

\subsection{Water activity and thermodynamic components of enantioselectivity}

A study of the temperature dependency of the enantioselectivity allowed the calculation of entropic and enthalpic contributions to the enantioselectivity towards butan-2-ol, pentan-2-ol and hexan-3-ol (Table 1). In the case of octan-4-ol, variation of enantioselectivity with temperature was too low to allow such a calculation.

Results showed that the entropic part of the differential activation free energy $\Delta_{\mathrm{R}-\mathrm{S}} \Delta G^{t}$ was very important for the kinetic resolution of the enantiomers. Indeed, butan-2-ol, pentan-2-ol and hexan-3-ol all had entropic terms $\left(\left|T \Delta_{\mathrm{R}-\mathrm{S}} \Delta S^{\hat{f}}\right|\right)$ that were between $45 \%$ and $194 \%$ of the differential activation free energy $\left(\left|\Delta_{\mathrm{R}-\mathrm{S}} \Delta G^{f}\right|\right)$.

Activation entropy is a complex term which is built up from differences in the degrees of freedom of the substrate and the enzyme, as well as contributions from selective release of solvent from the active site, upon binding of enantiomeric substrates [23,24]. In liquid systems, it is practically impossible to separate the contribution to $\Delta_{\mathrm{R}-\mathrm{S}} \Delta S^{t}$ related to differential solvation effects from other contributions. In the case of solid/gas catalysis however, and at aw close to 0 , the factors influencing activation entropy are restricted to rotational and translational freedom in the enzyme-substrate complex and the $\Delta_{\mathrm{R}-\mathrm{S}} \Delta S^{t}$ obtained can be considered as an intrinsic parameter independent of the microenvironment of the enzyme. This "intrinsic" entropic part obtained at a close to 0 was found to be about $50 \%$ of the differential activation free energy, for all alcohols tested: butan-2-ol, pentan-2-ol 
and hexan-3-ol. This shows that the contribution of "intrinsic" entropic phenomena to enantioselectivity was of significant importance. When water is added in the gaseous stream, one notes that the entropic component of $\Delta_{\mathrm{R}-\mathrm{S}} \Delta G^{t}$ increases. This can be explained by a differential trapping or release of water in the active site upon formation of the transition state.

\subsection{Adsorption studies}

The aim of the following part is to correlate the effects of water on CALB enantioselectivity with effective hydration state of the catalyst, as determined by different adsorption studies.

\subsubsection{Water sorption isotherm on immobilized CALB}

CALB immobilized on Chromosorb showed a linear adsorption isotherm up to $\mathrm{a}_{\mathrm{w}} 0.35$ and after that the isotherm became exponential (Figure 5). Similar behavior has been observed for other lipases $[25,26]$. The number of water molecules bound to CALB increased with 80 per molecule of protein, when the water activity increased from 0 to 0.2 . This number of water molecules covers only one fifth of the surface of the enzyme, which means that these water molecules had found specific binding spots. Even at $\mathrm{a}_{\mathrm{w}} 0.35$ only $50 \%$ of the protein surface was covered by newly adsorbed water molecules. At $\mathrm{a}_{\mathrm{w}} 0.9$ the number of water molecules was enough to cover the enzyme with several layers. As hydrolysis reached a maximum value just above $a_{w} 0.2$ it shows that the nucleophilic water has a specific affinity to the active site (Figure 2).

\subsubsection{Water activity and heat of sorption of pentan-2-ol on immobilized lipase}

The heat of sorption is a direct measure of the strength of interaction between the surface of a solid and gas molecules adsorbed on the surface, which is clearly influenced by $\mathrm{a}_{\mathrm{W}}$. Indeed, 
when increasing humidity, water molecules are able to progressively mask polar surface groups, leading to a decrease of the absolute value of adsorption enthalpy $(|\Delta H a d s|)$, as long as the relative humidity becomes high enough to mask all polar groups.

The specific binding of pentan-2-ol as a function of $\mathrm{a}_{\mathrm{w}}$ was measured by IGC, which affords adsorption enthalpy $\Delta H_{a d s}$ (Figure 6). It can be seen that the interaction with the enzyme went down rapidly from $\mathrm{a}_{\mathrm{w}} 0$ to 0.2 followed by a less pronounced decrease for $\mathrm{a}_{\mathrm{w}}$ above 0.2 . In this second part of the curve, the retention time of the alcohol on immobilized CALB measured by IGC, decreased significantly and presented less variation with temperature. Consequently $|\Delta H a d s|$ stabilised, and this could be attributed to the fact that water molecules adsorbed on the immobilized enzyme, hid high energy sites from pentan-2-ol.

The change in $\mathrm{a}_{\mathrm{w}}$ dependence coincides with that of the optimum for enantioselectivity (Figure 4). The two observations strengthen the hypothesis that water binding to the specificity pocket competes with pentan-2-ol. For $\mathrm{a}_{\mathrm{w}}$ below 0.2 , water acts mostly as an enantioselective inhibitor, through specifically located effects, by binding in the stereospecificity pocket. Above this point, water acts predominantly as a "solvent", with high entropic effects. This can also be connected to the large entropic term seen for enantioselectivity at high water activity. To make room for the alcohol several water molecules that were trapped at the enzyme left and gained motional freedom.

\section{Conclusion}

In this paper, we have demonstrated that water had a pronounced effect on CALB enantioselectivity towards secondary alcohols, as soon as they have the structural features for being well-resolved by this enzyme. The mechanism by which water favours or disfavours enantioselectivity strongly depends on hydration state of the catalyst, as determined by IGC 
technique. At low hydration, the role as enantioselective inhibitor is predominant, whereas when full coverage of polar groups of enzyme by water is obtained, the main effect arises from entropic phenomena.

\section{Acknowledgements}

This work was supported by the French Ministry of National Education, Higher Education and Research and the Swedish Research Council. Dr Majid Naderi (Surface Measurement Systems Ltd., London, U.K.) is thanked for water adsorption isotherm measurements of Chromosorb alone and with immobilized lipase.

\section{References}

[1] R.J. Kazlauskas, A.N.E. Weissfloch, A.T. Rappaport and L.A. Cuccia, J. Org. Chem. 56 (1991) 2656-2665.

[2] D. Rotticci, F. Haeffner, C. Orrenius, T. Norin, K. Hult, J. Mol. Catal. B: Enzym. 5 (1998) 267-272.

[3] B.Y. Hwang, H. Scheib, J. Pleiss, B.G. Kim, R.D. Schmid, J. Mol. Catal. B: Enzym. 10 (2000) 223-231.

[4] T. Schulz, J. Pleiss, R.D. Schmid, Protein Sci. 9 (2000) 1053-1062.

[5] J. Ottosson, L. Fransson, J.W. King, K. Hult, Biochim. Biophys. Acta 1594 (2002) $325-334$

[6] A. Ducret, M. Trani, R. Lortie, Enzyme Microbial. Technol. 22 (1998) 212-216.

[7] E. Wehtje, D. Costes, P. Adlercreutz, J. Mol. Catal. B: Enzym. 3 (1997) 221-230.

[8] M. Persson, D. Costes, E. Wehtje, P. Adlercreutz, Enzyme Microbial. Technol. 30 (2002) 916-923. 
[9] A. Jönsson, E. Wehtje, P. Adlercreutz, B. Mattiasson, Biochim. Biophys. Acta 1430 (1999) 313-322.

[10] R.S. Phillips, Enzyme Microbial. Technol. 14 (1992) 417-419.

[11] R.S. Phillips, Trends Biotechnol. 14 (1996) 13-16.

[12] V. Léonard, L. Fransson, S. Lamare, K. Hult, M. Graber, ChemBioChem 8 (2007) $662-667$

[13] S. Lamare, M.D. Legoy, Biotechnol. Bioeng. 45 (1995) 387-397.

[14] A.V. Kiselev, I. Yashin, Gas Adsorption Chromatography, Plenum Press, New York, 1969.

[15] J.R. Condor, C.L. Young, Physicochemical Measurement by Gas Chromatography, John Wiley and Sons, Chichester, 1979.

[16] J.C. Rotticci-Mulder, M. Gustavsson, M. Holmquist, K. Hult, M. Martinelle, Protein Expression Purif. 21 (2001) 386-392.

[17] V. Léonard, , S. Lamare, M.D. Legoy, M. Graber, J. Mol. Catal. B: Enzym. 32 (2004) 53-59.

[18] H.E. Newell, G. Buckton, D.A. Butler, F. Thielman, D.R. Williams, Int. J. Pharm. 217 (2001) 45-56.

[19] J. Uppenberg, N. Ohrner, M. Norin, K. Hult, G.J. Kleywegt, S. Patkart, V. Waagen, T. Anthonsen, T.A. Jones, Biochemistry 34 (1995) 16838-16851.

[20] M.P. Bousquet-Dubouch, M. Graber, N. Sousa, S. Lamare, M.D. Legoy, Biochim. Biophys. Acta 1550 (2001) 90-99.

[21] M. Graber, M.P. Bousquet-Dubouch, S. Lamare, M.D. Legoy, Biochim. Biophys. Acta, Proteins Proteomics 1648 (2003) 24-32.

[22] J. Ottosson, L. Fransson, K. Hult, Protein Sci. 11 (2002) 1462-1471. 
[23] P.L.A. Overbeeke, J. Ottosson, K. Hult, J.A. Jongejan, J.A. Duine, Biocatal. Biotransform. 17 (1999) 61-79.

[24] R.S. Phillips, J. Mol. Catal. B: Enzym. 19 (2002) 103-107.

[25] Y. Caro, M. Pina, F. Turon, S. Guilbert, E. Mougeot, D.V. Fetsch, P. Attwool, J. Graille, Biotechnol. Bioeng. 77 (2002) 693-703.

[26] R.M. de la Casa, J.M. Guisan, J.M. Sanchez-Montero, J.V. Sinisterra, Enzyme Microbial. Technol. 30 (2002) 30-40. 